\title{
Predicting our own and others' future preferences: The role of social distance
}

\begin{tabular}{|r|l|}
\hline Journal: & Quarterly Journal of Experimental Psychology \\
\hline Manuscript ID & QJE-STD 17-127.R2 \\
\hline Danuscript Type: & Standard Article \\
\hline Complete List of Authors: & $\begin{array}{l}\text { Bauckham, Grace; University of East Anglia } \\
\text { Lambert, Rachel; University of East Anglia, School of Psychology } \\
\text { Atance, Cristina; University of Ottawa, } \\
\text { Davidson, Patrick; University of Ottawa, } \\
\text { Taler, Vanessa; University of Ottawa } \\
\text { Renoult, Louis; University of East Anglia, }\end{array}$ \\
\hline \hline Keywords: & Future thinking, Projection bias, Presentism bias, Self, Close other, Aging \\
\hline & \multicolumn{2}{|l}{} \\
\hline
\end{tabular}

SCHOLARONE

Manuscripts 


\section{Predicting our own and others' future preferences: The role of social distance}

Grace Bauckham ${ }^{1}$, Rachel Lambert ${ }^{1}$, Cristina M. Atance ${ }^{2}$, Patrick S.R. Davidson ${ }^{2}$, Vanessa Taler $^{2}$, Louis Renoult ${ }^{1}$

${ }^{1}$ University of East Anglia, UK

${ }^{2}$ University of Ottawa, Ontario, Canada

Submitting Author:

Renoult Louis

University of East Anglia

Norwich

UK 


\begin{abstract}
People underestimate how much their preferences will change in the future, a phenomenon sometimes referred to as a "presentism bias". Recently we found that this presentism bias is attenuated when thinking about the preferences of other people. The aim of the current study was to investigate whether predicting future preferences also differs depending on the level of social distance between self and other. Sixty-seven participants completed a perspective-taking task in which they were required to think about their own preferences, those of a generic peer and those of a close other both now and in the future. They were also asked to consider the preferences of an older adult now. Participants predicted less change between their current and future preferences than between the current and future preferences of a generic peer. Predicted change in preferences for a close other were similar, but not identical, to those made for the self. When considering relevant future preferences, participants predicted less change for themselves than for their close others, and less change for close others than for generic peers. In other words, as social distance increases, the presentism bias decreases. Interestingly, participants estimated that both they and their peers would not change so much that they become similar to current older adults. Simulating the future perspectives of a generic peer or, even better, the current perspectives of an older adult, may thus result in improved long-term decision-making, as it may enable a more realistic estimation of the magnitude of likely changes in the future.
\end{abstract}

Keywords: Future thinking, Projection bias, Presentism bias, Self, Close other, Aging 


\section{Introduction}

Thoughts about the future occupy a prominent position in the mental life of humans (Atance, 2008; D'Argembeau, Renaud, \& Van der Linden, 2011), with some studies reporting twice as many future-oriented as past-oriented thoughts (Jason, Schade, Furo, Reichler, \& Brickman, 1989). Future thinking can be directed towards ordinary mundane events, such as what to have for lunch tomorrow, but it can also be directed towards more significant life choices, such as getting married or accepting a specific job, that will impact us in a more long-term way. Unfortunately the conclusions that we draw about the future are not always accurate, and people typically underestimate how much their future preferences and values will change over time (Gilbert \& Wilson, 2007; Loewenstein, O'Donoghue, \& Rabin, 2003; Quoidbach, Gilbert, \& Wilson, 2013). Several terms have been proposed to explain this phenomenon. Loewenstein et al. (2003) describe a "projection bias" by which we project our current preferences, feelings or values into the future, despite the fact these may no longer be relevant. Quoidbach et al. (2013) discussed an "end of history illusion" by which adults seem to believe that the amount they are changing has slowed down, and they have become the person that they will remain for the rest of their lives. Another similar mechanism described by the same authors is the "presentism bias", which is the proneness to interpret past and future versions of one's self in relation to one's present motives and knowledge (Cameron, Wilson, \& Ross, 2004; Gilbert, Gill, \& Wilson, 2002). These terminologies all constitute different levels of explanations of the same phenomenon: underestimation of change of preferences and values in the future. However, for cohesiveness, throughout the manuscript we will use the term "presentism bias."

The existence of presentism biases demonstrates that people have difficulties when making predictions about the future. Recently, Renoult and colleagues (Renoult, Kopp, 
Davidson, Taler, \& Atance, 2016) investigated whether these biases appear exclusively when people consider their own future preferences or whether they also characterize the predictions they make for others. We used a perspective-taking task in which young adults (18-33 years old) were asked how much they like stereotypically-young person things (e.g., top 40 music, adventure vacations) and stereotypically-old person things (e.g., jazz, playing bridge) now and how much they would like them in the future, when aged 70 . Participants also estimated these same preferences for a same-sex generic peer. Finally, they also rated how much an older adult, aged 70, currently liked these items.

Participants predicted less change between their own current and future preferences than between the current and future preferences of a peer. This suggests that the presentism bias is attenuated when making predictions for others. This finding is reminiscent of a reduction in other cognitive biases when thinking about others, such as the optimism bias (Baker \& Emery, 1993; Grysman, Prabhakar, Anglin, \& Hudson, 2013) or the temporal discounting bias (Pronin, Olivola, \& Kennedy, 2008). People thus seem more rational or adaptive in their thinking when reasoning about others, an effect referred to as the "beneficial other effect" by Lee and Atance (2016).

One explanation for this effect is that the psychological distance imparted by considering someone else's perspective allows us to think more abstractly and thus in a more meaning-based than experience-based manner (Liberman, Trope, \& Stephan, 2007). This, in turn, allows us to escape the "here-and-now" and the more emotional and visceral aspects of decision-making (Bechara \& Damasio, 2005; Loewenstein, 1996; Naqvi, Shiv, \& Bechara, 2006). Interestingly, in the Renoult et al. (2016) study, even though the presentism bias was indeed attenuated when making predictions for a peer, this bias was not abolished. Participants judged that the preferences of their peers would not change so much in the 
future as to be similar to those of a current older adult. Thus, making predictions for a generic peer, even if less influenced by current desires and impulses, may still rely on unrepresentative or decontextualized future simulations (Gilbert \& Wilson, 2007), perhaps reflecting inherent limits in our future thinking capacities.

One important aspect of the design used in Renoult et al. (2016) is that participants considered the preferences of a generic unknown peer. The literature on social distance suggests that other cognitive biases, like the optimism bias (Grysman et al., 2013) and the temporal discounting bias (Kim, Schnall, \& White, 2013; Ziegler \& Tunney, 2012), are more similar for self and close others as compared to more socially distant others. Accordingly, we can hypothesize that presentism biases when evaluating one's own and a close other's future preferences are similar and, importantly, are attenuated when considering those of a generic peer. Such potential differences in how people think about their own, close others', and more distant others' futures are important for at least several reasons. First, our common misconceptions about future changes can have severe consequences, such as making poor health or financial choices in the present that our future selves will regret. In the case of taking the perspective of close-others, research shows that we often misjudge their preferences and make decisions that are contrary to their wishes (Tunney \& Ziegler, 2015). Consequently, identifying methods to reduce biases that people experience when predicting their future preferences and values may have far-reaching impact.

Interestingly, a number of studies have shown that the content of future predictions can be modified. For instance, performing detailed or repeated simulations of future events may increase their subjective plausibility as well as the number of details produced (Sherman, Cialdini, Schwartzman, \& Reynolds, 1985; Szpunar \& Schacter, 2013). Although there exist several methods to change the way that people think about the future, to our 
knowledge, these methods have not targeted how perspective and social distance, more specifically, may impact the accuracy of people's predictions about their future preferences. It is thus important to better understand whether taking other people's perspectives (of different social distance or different ages) may help us to optimize the accuracy and adaptiveness of our decisions.

The aim of the present study was to investigate whether the presentism bias varies as a function of social distance. We used a similar perspective-taking paradigm as in Renoult et al. (2016), in which we asked young adults how much they liked stereotypically-young person and stereotypically-old person things now and how much they would like them in the future. They also had to predict how much a generic peer and a close other (friend or romantic partner) would like these items now and in the future. Finally, participants had to estimate how much a 70-year-old adult liked these items now. We hypothesized that the use of this paradigm would produce a graded presentism bias such that participants would estimate that their own preferences would change less in the future than those of a close other, and that the preferences of a close other would change less than those of a generic peer. We also predicted that self and peers' preferences (both close other and generic other) would not change so much in the future as to be similar to those of a current older adult, reflecting a persistence of the presentism bias and a general difficulty in predicting change. 


\section{Methods}

Participants

Sixty-seven young adults (53 females; mean age $=20.11$ years, range $=18-27$ ) participated in the perspective-taking task. They were recruited through the School of Psychology research participation system at the University of East Anglia and via social media. All participants signed an online informed consent form approved by the ethics committee of the school of Psychology.

\section{Perspective-taking task}

Selection of stimuli

Stimuli were based on those used in Renoult et al. (2016); however, some items were altered from a Canadian to a UK version (e.g., Sears department store was changed to Marks and Spencer's department store; see appendix for the list of stimuli). We selected the stimuli by asking 25 participants who did not participate in the experiment (13 females; mean age $=21.56$, range $=19-25)$ how much they liked 20 stereotypically-young-person things (e.g., rollerblading, going to festivals) and 20 stereotypically-old-person things (e.g., birdwatching, gardening), now, and how much they would like these in the future at age 70. In each trial, the participants responded using a 7-point Likert scale: 1-strongly dislike, 2dislike, 3-dislike somewhat, 4-neutral, 5-like somewhat, 6-like, 7-strongly like. We then calculated the average difference in ratings between the now and future categories for each item and selected the 10 stereotypically-young-person and 10 stereotypically-old-person items that differed most in ratings according to time (now versus future). Paired-samples t- 
tests were used to confirm that all selected items differed significantly between "now" and "future" ratings (all $p s<0.01$ ).

Task design

The combined total of 20 stereotypically-young and old-person items provided the stimuli for the perspective taking task (see appendix). The same 7-point Likert scale as in the norming study was used (from 1-Strongly dislike to 7-Strongly like, see above). The questions were presented in the form of an online questionnaire created using the online survey software Qualtrics (https://www.qualtrics.com/). We asked participants how much they liked stereotypically-young-person things (e.g., rollerblading, going to festivals) versus stereotypically-old-person things (e.g., birdwatching, gardening) now, and how much they would like them when they are 70 years old. We also asked them to rate these items for a same-age peer, a close other (friend or romantic partner) and an older adult. For the close other condition, participants were asked whether they had been in a relationship for at least 6 months. If yes, they were instructed to think about this person to complete the close other condition. If not, they had to think of someone they considered to be a close friend ${ }^{1}$. In trials in which participants were asked to take the perspective of a generic peer, the photograph of an unknown gender-matched face was presented as "Someone else your age". In trials in which participants were asked to take the perspective of an older adult, the photograph of an unknown gender-matched face was presented as "a 70-year-old". In the close other condition, the name provided by the participant was presented. There were four perspectives (self, generic peer, close other, older adult), and 2 times (now versus future) that corresponded to seven separate blocks of trials: Self-Now, Self-Future, Generic PeerNow, Generic Peer-Future, Close other-Now, Close other-Future, Older adult-Now. The

\footnotetext{
${ }^{1} 30$ participants thought about a close friend and 37 about a romantic partner. Results were similar for both categories, but are not broken down by category due to low statistical power.
} 
blocks were presented randomly and each of the stereotypically-old and young items were also presented randomly within each of the blocks. Each participant answered a total of 140 questions regarding preferences.

\section{Statistical analyses}

To test the extent to which ratings of one's own and peers' preferences were similar/different, we conducted a repeated-measures ANOVA on preference ratings with the following factors: Time (now versus future), Item type (stereotypically-young versus stereotypically-old person things) and Perspective (self, generic peer, close other). In all cases, the dependent variable was preference scores.

To test the extent to which predictions of self and peers' preferences would differ from an older adult's perspective, we conducted two additional repeated-measures ANOVAs. These ANOVAs included Perspective (self, generic peer, close other, older adult) and Item type (stereotypically-young versus stereotypically-old-person things) as withinsubject factors (time was not included because the older adult was only asked about in the now condition). One of these analyses was conducted with the Now conditions, and the other with the Future conditions of self and peers.

Partial eta-squared $\left(\eta_{p}^{2}\right)$ is indicated as a measure of effect size in all analyses. 


\section{Results}

\section{Main Analysis}

The repeated-measures ANOVA on preference ratings revealed main effects of perspective, $F(2,130)=5.880, M S E=0.535, p=.005, \eta p^{2}=.083$, and time $F(1,65)=$ 180.374, MSE $=0.483, p<.001, \eta p^{2}=.735$. A three-way interaction between time, item type and perspective was also identified $F(2,130)=29.421, M S E=0.629, p<.001, n p^{2}=$ .312. We followed up these results with separate analyses of preference ratings by time period and by item type.

\section{Analysis by Time Period}

Now condition: Analysis for the now condition showed a main effect of perspective $F(2,130)=4.402, M S E=0.404, p=.019, n p^{2}=.063$, and item type $F(1,65)=155.225, M S E=$ $1.515, p<.001, \eta p^{2}=.705$, as well as an interaction between perspective and item type $F(2$, $130)=8.142, M S E=0.77, p=.001, n p^{2}=.111$. Pairwise comparisons for stereotypicallyyoung items revealed that participants attributed higher preference ratings to a generic peer $($ mean $=5.52)$ in comparison to themselves $($ mean $=5.04 ; p=.001)$, and in comparison to a close other (mean $=5.03 ; p=.001$ ). No significant difference was found between the preference ratings for self and close other $(p=.975)$. In contrast, for the stereotypically-old items, participants gave higher preference ratings to self (mean $=3.90$ ) than to a generic peer $($ mean $=3.52 ; p=.001)$, or to a close other $($ mean $=3.55 ; p=.002)$. However, ratings for close other and generic peer did not significantly differ $(p=823)$.

This analysis about current preferences therefore suggests that participants rated generic peers as liking "young" items currently the most in comparison to the self and close 
others. In contrast, participants judged that they, themselves, currently liked "old" items more than generic peers and close others (see Figure 1).

Future condition: Analysis for the future condition again revealed a main effect of perspective $F(2,130)=7.423, M S E=0.356, p=.001, \eta p^{2}=.102$, and item type $F(1,65)=$ 159.533, MSE $=1.96, p<.001, \eta p^{2}=.711$. An interaction between perspective and item type was also identified $F(2,130)=10.724, M S E=1.242, p<.001, n p^{2}=.142$. Pairwise comparisons for stereotypically-young items revealed that participants allocated higher ratings to a close other $($ mean $=3.03)$ in comparison to a generic peer $($ mean $=2.67 ; p=$ .031). No significant difference was found when comparing close other and self (mean = 2.90; $p=.384)$, and the self and generic peer $(p=.173)$. In contrast, for the stereotypicallyold items, participants attributed higher ratings to a generic peer (mean $=5.16)$ than the self $($ mean $=4.38 ; p<.001)$, and the close other (mean $=4.42 ; p<.001)$. No significant difference was found between the preference ratings of the close other and the self $(p=$ .787).

This future preferences analysis therefore indicates that participants predicted they would like "young" items similarly to close others and generic peers. However, they judged that they and their close others would like "old" items less than generic peers would (see Figure 1).

\section{Analysis by Item Type}

Stereotypically-young items: Analysis of preference ratings for stereotypically-young items revealed a main effect of time $F(1,65)=354.105, M S E=1.509, p<.001, \eta p^{2}=.845$, and an interaction between perspective and time $F(2,130)=13.087, M S E=0.502, p<.001$, 
$\eta p^{2}=.168$. Pairwise comparisons showed that, as expected, young items were preferred now relative to the future for both the self (means $=5.04$ vs 2.90 , respectively; $p<.001$ ), for a generic peer $($ means $=5.52$ vs $2.67 ; p<.001)$, and a close other $($ means $=5.03$ vs $3.03 ; p<$ .001). Importantly, however, the difference in ratings was greater for a generic peer (mean difference $=2.86$ ) as compared to the self (mean difference $=2.13 ; p<.001$ ), or a close other $($ mean difference $=2.00 ; p<.001)$. There was no significant difference between the ratings for the self and the close other $(p=.422)$.

This analysis of change in preferences over time for "young" items revealed than participants predicted less change in preferences for self and close other than for a generic peer (see Figure 2).

Stereotypically-old items: Analysis of preference ratings for stereotypically-old items revealed main effects of perspective $F(2,130)=5.023, M S E=0.767, p=.008, n p^{2}=.072$, and time $F(1,65)=87.680, M S E=1.120, p<.001, n p^{2}=.574$, as well as an interaction between perspective and time, $F(2,130)=40.333, M S E=0.307, p<.001, \eta p^{2}=.383$. Again, as expected, pairwise comparisons highlighted that old items were rated as preferred in the future relative to now for the self (means $=4.38$ vs 3.90 , respectively; $p<.001$ ), for a generic peer (means $=5.16$ vs $3.52 ; p<.001$ ), and for a close other (means $=4.42$ vs $3.55 ; p<.001$ ). The difference in ratings with time was greatest for a generic peer (mean difference $=1.64$ ) as compared to the self (mean difference $=0.48 ; p<.001$ ), or as compared to a close other (mean difference $=0.9 ; p<.001$ ). Moreover the difference in ratings was greater for a close other than for the self $(p=.001)$.

This analysis of change of preference over time for "old" items suggests that participants were predicting less change for themselves and their close others than for 
generic peers. However, they also predicted less change for themselves than for their close others (see Figure 2).

\section{Comparison of younger and older adults' perspectives}

Comparison between self, peers, and older adult "now".

To test whether participants judged that they, their peers and their close others would have higher preference ratings for the young items and lower preference ratings for old items now than an older adult would, we ran a repeated-measures ANOVA comparing older adults, self, generic peers and close others in the 'now' condition. This analysis revealed a main effect of item type $F(1,65)=12.452, M S E=1.005, p=.001, \eta p^{2}=.159$, and a main effect of perspective $F(3,195)=46.97, M S E=0.46, p<.001, \eta p^{2}=.416$. An interaction between perspective and item type also emerged, $F(3,195)=258.895, M S E=$ $1.109, p<.001, \eta p^{2}=.78$.

Stereotypically-young items: For the stereotypically-young items, there was a main effect of perspective $F(3,195)=257.577$, MSE $=0.69, p<.001, n p^{2}=.796$. Pairwise comparisons revealed that the participants gave lower preference ratings to an older adult $($ mean $=2.02)$ than the self $($ mean $=5.04 ; p<.001)$, close other $($ mean $=5.03 ; p<.001)$, and generic peer $($ mean $=5.52 ; p<.001)$.

Stereotypically-old items: For the stereotypically-old items there was a main effect of perspective $F(3,195)=89.273, M S E=0.76, p<.001, n p^{2}=.575$. Pairwise comparisons showed that, for these items, participants attributed higher preference ratings to an older 
adult $($ mean $=5.42)$, than to a generic peer $($ mean $=3.52 ; p<.001)$, a close other (mean $3.55 ; p<.001)$ and the self $($ mean $=3.90 ; p<.001)$.

As expected, these analyses indicated that participants judged that older adults currently liked "young" items less and "old" items more than themselves, close others and generic peers (see Figure 1).

Comparison between older adult "now" and self and peers in the "future"

To determine whether the extent of the predicted change of preferences for the self, generic peer and close other in the 'future' condition would be comparable to those of an older adult 'now', we ran a repeated-measures ANOVA comparing older adults "now" with close other, generic peer and self in the future condition. This analysis revealed that there was a main effect of perspective $F(3,195)=5.684, M S E=0.362, p=.002, \eta p^{2}=.079$, and a main effect of item type $F(1,65)=296.004, M S E=2.17, p<.001, \eta p^{2}=.818$. An interaction between perspective and item type was also found, $F(3,195)=32.78, M S E=1.153, p<.001$, $\eta p^{2}=.332$

Stereotypically-young items: For the stereotypically-young items there was a main effect of perspective $F(3,195)=18.748, M S E=0.823, p<.001, \eta p^{2}=.221$. Pairwise comparisons showed that, for these items, participants gave the older adult (mean $=2.02$ ) a lower preference rating in comparison to the self (mean $=2.91 ; p<.001)$, a close other (mean $=$ $3.03 ; p<.001)$, and a generic peer $($ mean $=2.67 ; p<.001)$.

Stereotypically-old items: For the stereotypically-old items a main effect of perspective was found $F(3,195)=36.132, M S E=0.582, p<.001, \eta p^{2}=.354$. Pairwise 
comparisons revealed that, for these items, participants gave higher preference ratings to an older adult (mean $=5.42)$, than they did to a generic peer (mean $=5.16 ; p=.035)$, a close other $($ mean $=4.42 ; p<.001)$, and the self $($ mean $=4.38 ; p<.001)$.

Considering these results together shows that even though participants predicted that their preferences and those of generic peers and close others would change over time, they would still not change enough to be similar to the current preferences of an older adult.

\section{Control Analyses}

\section{Item level analysis}

The difference in mean ratings for now versus future judgments might be similar between two conditions (e.g., self versus close other) and yet the amount of changes in preference might not be equivalent if some items are associated with increases in ratings while other items are associated with decreases. We thus computed an index of change based on responses given to the same items for now versus future judgments to verify that the presentism bias was homogeneous across individual items. For this, we subtracted preference scores for each item and each participant; for stereotypically-young items, we computed a "future minus now" score and for stereotypically-old items, a "now minus future" score. We then ran a repeated-measures ANOVA with the following within-subject factors: individual item (10 levels corresponding to 10 individual items, see appendix), item type (2 levels: stereotypically-young versus stereotypically-old items) and perspective (self, generic peer and close other).

This analysis revealed main effects of individual item, $F(9,576)=18.88, M S E=3.928$, $p<.001, n p^{2}=.23$, item type $F(1,64)=174.16, M S E=9.846, p<.001, n p^{2}=.73$, and 
perspective $F(2,128)=28.73, M S E=12.809, p<.001, \eta p^{2}=.31$, as well as interactions between item type and perspective, $F(2,128)=5.43, M S E=4.364, p=.006, \eta p^{2}=.08$, and item type and individual item, $F(9,576)=6.81 \mathrm{MSE}=2.768, p<.001, \eta p^{2}=.096$, but no interaction between item type, perspective and individual item, $F(18,1152)=1.53, M S E=$ $2.131, p=.102, n p^{2}=.02$.

Pairwise comparisons on the stereotypically-young items showed main effects of perspective, $F(2,132)=14.03, M S E=10.298, p<.001, \eta p^{2}=.17$, and individual item, $F(9$, $594)=17.94, M S E=3.303, p<.001, n p^{2}=.21$, but no interaction between these factors, $F(18,1188)=0.79, M S E=2.213, p=.67, n p^{2}=.01$. This indicated that the effect of perspective was homogeneous across individual items for the stereotypically-young items.

For the stereotypically-old items, there were again main effects of perspective $F(2$, $132)=39.57$, MSE $=6.192, p<.001, \eta p^{2}=.38$, and individual item $F(9,594)=9.87$, MSE $=$ $3.463, p<.001, \eta p^{2}=.13$, but also an interaction between these factors, $F(18,1188)=2.81$, MSE $=2.144, p=.001, n p^{2}=.04$. Pairwise comparisons for each stereotypically-old item revealed that the effect of perspective was significant for all items $(p<0.02)$, except for the item cruise holidays for which the effect was only marginal $(p=0.09)$. Importantly, for all items, descriptive statistics indicated the same pattern of difference between current and future preferences: the magnitude of change in ratings was greater for peer followed by close other followed by self (peer > close other > self), as seen in the analyses using average preferences across items (see above). Therefore, even though one of the 10 stereotypicallyold items showed a less significant difference between current and future preferences, the effect of perspective was remarkably similar across individual items.

\section{Preferences of a control group of older adults}


In the current experiment, we asked a group of young people to estimate their own current and future preferences (when they are 70) and the current preferences of a 70-yearold. However, these predictions were based on what participants imagine an older adult to be like, rather than the actual reports of older adults. It is thus unclear whether the presentism bias that we observed reflects an inaccurate prediction of future change (i.e., participants thinking that they will not change enough to be similar to current older adults) or an inaccurate vision of what 70-year-olds like (e.g., participants underestimating the extent to which older adults may like young people items).

We thus ran a complementary analysis with a group of 29 older adults (16 Females; mean age $=69.83$, range $65-80)$. Using the same instructions as for our group of younger adults, we asked about their current preferences (Now condition), but also about their preferences when they were 20 years old (Past condition, mirroring our group of 20-yearolds who were asked about when they are 70). For these analyses, we removed the item Instagram, which did not exist 50 years ago. We ran a repeated-measures ANOVA with the following within-subject factors: item type (2 levels: stereotypically-young versus stereotypically-old items) and time (now versus past).

This analysis revealed an interaction between item type and time, $F(1,28)=60.82$, MSE $=0.285, p<.001, n p^{2}=.69$. Pairwise comparisons revealed that older adults currently preferred stereotypically-old items $($ mean $=4.11)$ to stereotypically-young items (mean = 2.77; $p<.001)$. Analysis by time period showed higher ratings for stereotypically-young items at age 20 (mean $=3.82)$ than currently at age 70 (mean $=2.77 ; p<.001)$, and for stereotypically-old items at age $70($ mean $=4.11)$ than at age $20($ mean $=3.59 ; p<.001)$.

This control analysis indicated that our stereotypically-young and -old items appropriately reflected the current and past preferences of older adults (i.e., they currently 
preferred "old" items to "young" items). Moreover, the actual preferences of older adults were similar to those estimated by our group of young adults for a generic older adult. Our young participants' estimations were thus accurate, even though they reported that their own preferences would not change so much as to make them similar to those of older adults.

\section{Discussion}

Research consistently shows that we tend to mispredict our future preferences and values, and typically underestimate how much we will change - a phenomenon referred to as a "presentism bias" (Cameron et al., 2004; Gilbert et al., 2002). We recently found that this presentism bias is attenuated when we consider the future preferences of a same-sex peer (Renoult et al., 2016). The aim of the present study was to test whether the presentism bias also varies with social distance. Our participants performed a perspective-taking task in which they had to consider their own preferences, those of a generic peer and those of a close other now and in the future (i.e., when they are 70). Participants also had to estimate the current preferences of an older adult. We will briefly review our main results and then discuss these findings in the context of the literature on perspective-taking biases.

Participants predicted less change between their own current and future preferences than between the current and future preferences of a generic peer, replicating the findings from Renoult et al. (2016). In addition, we found that participants predicted less change for a close other's preferences than for those of a generic peer. These results suggest that similar presentism biases exist when we consider our own preferences and those of our close others. Consistent with this observation, when considering currently 
relevant preferences (i.e., stereotypically-young items such as "rollerblading" and "going to festivals"), ratings for self and close others did not differ. However, even though preference ratings for a close other were generally found to be similar to those made for the self, they were not identical. When considering preferences relevant in the future (i.e., stereotypically-old items such as "birdwatching" and "gardening"), participants predicted less change for themselves than for their close others, and less change for close others than for generic peers, showing a graded increase in predicted change between self, close others and generic peers.

Consistent with Renoult et al. (2016) and previous studies (reviewed in Loewenstein et al., 2003), our participants did not have a strict "end of history illusion" (Quoidbach et al., 2013). They estimated that they, their generic peers and close others currently preferred stereotypically-young items and would prefer stereotypically-old items in the future. Nonetheless, as compared to generic peers, they showed a robust presentism bias by estimating that their preferences would change less in the future. These findings replicate those of Renoult et al. (2016) with a UK sample of participants (rather than a Canadian one) and a modified item list.

Interestingly, similar to our previous study, participants' underestimation of change over time was not only produced by lower ratings of "old items" in the future. As compared to generic peers, participants also judged that they currently liked "old" items more, and "young" items less. These results are reminiscent of the "false uniqueness bias" (reviewed in Chambers, 2008). False uniqueness is the tendency to think we are better or less typical than average. The items most relevant to the present (i.e., the stereotypically-young ones) were rated as currently more preferred by a generic peer than the self. In contrast, those relevant in the future, the stereotypically-old items, were rated as more preferred by the 
self now but more preferred by a generic peer in the future. This indicates that participants perceived themselves to be less typical than average for both types of items, and that false uniqueness biases are similar for judgements related to the present and the future (Kanten \& Teigen, 2008; see also Renoult et al., 2016).

Unlike the findings for generic peers, and in line with our hypotheses, preference ratings for self and close others were found to show many similarities. When considering currently-relevant preferences (i.e., stereotypically-young items), ratings for self and close others did not differ. As compared to themselves and their close others, participants estimated that the preferences of generic peers would change more over time. Thus, for currently-relevant preferences, presentism biases appear similar between self and close others. In contrast, when considering preferences relevant in the future (i.e., stereotypically-old items), participants predicted less change for themselves than for their close others, and less change for close others than for generic peers, showing a graded increase in predicted change (and a graded reduction in the presentism bias) between self, close other and generic peer. More specifically, differences between self and close others were due to higher current preference ratings for self than close others for stereotypicallyold items (ratings did not differ in the future condition). These results therefore suggest that, for preferences relevant in the future, presentism biases differ between self and close others.

Taken together, our results show that, for currently-relevant preferences, presentism biases are non-distinguishable when considering our own preferences and those of our close others, but are reduced when thinking about generic peers. In contrast, for preferences relevant in the future, presentism biases are maximal when considering our own preferences, reduced when taking the perspective of our close others and further 
reduced when thinking about generic peers. The "beneficial other effect" (Lee \& Atance, 2016), by which we typically make more adaptive choices when reasoning about others, may thus be even more prevalent for preferences relevant in the future. In contrast, for currently-relevant preferences, considering a close other's perspective may not allow enough psychological distance to escape our presentism biases. Interestingly, even though presentism biases were minimal when considering the perspective of generic peers, participants estimated that these peers would still not change so much as to be similar to current older adults. More specifically, they estimated that older adults currently liked young items less and old items more than any of the young adult groups would in the future. Importantly, this finding is not likely to be due to our participants' erroneous estimation of what older adults may like. Indeed, a control analysis with a group of older adults revealed that they currently liked old items more and young items less than they had at age 20. Presentism biases, while clearly attenuated when taking a generic peer perspective, may thus not be abolished.

Another interpretation of these findings is the presence of a generation effect, by which young adults might anticipate that they will not necessarily like the same things in the future as current older adults do now. However, a generational change in preferences would likely have resulted in a global decrease in future ratings for all items compared to current ratings for older adults. This is because as yet unknown "new" items would be preferred by the next generations. In contrast, our participants estimated that they and their peers would like stereotypically-young items more and stereotypically-old items less in the future, as compared to current older adults. Therefore, unless one speculates that a generation effect would only apply to one set of items, our findings are more easily 
interpretable as an underestimation of change or, presentism bias (e.g., participants thinking that they will still like young items in the future), than as a generation effect.

An important factor when considering social distance and comparing self, generic peers and close others is the qualitative gap that exists between self-relevant and non-selfrelevant stimuli. As pointed out by Sui \& Humphreys (2015), a recurring question in research on the self is whether self-reference effects are influenced by other factors, such as enhanced familiarity of the stimulus, enhanced reward value, or increased emotional valence, as compared to non-self-relevant stimuli. Even though we believe, like these authors, that the specificity of self-representations cannot be completely reduced to these factors, they are nonetheless inherent to how we typicality operationalise the concept of self. Accordingly, we would have more knowledge about ourselves and our close others than about generic peers, and this knowledge should also have increased emotional valence. These factors could thus influence effects of social distance and comparisons between self, close others and generic peers.

Why then would social distance (i.e., thinking about a generic peer rather than a close one) or difference in age (i.e., a young individual considering the perspective of an older adult) help us overcome presentism biases? One possibility is that the psychological distance imparted by considering someone else's perspective allows us to more efficiently escape the here-and-now and the more emotional and visceral aspects of decision-making (Bechara \& Damasio, 2005; Loewenstein, 1996; Naqvi et al., 2006). Accordingly, we may not always be in the best position to make decisions for a close other because we might be too "contaminated" by our own affective states (which could thus result in similar biases). Psychological distance results in thinking more abstractly (Liberman et al., 2007) and thus taking a generic peer perspective may reduce the presentism bias by relying more on 
general semantic knowledge about the world and schemas (i.e., what people typically do), as compared to more difficult predictions based on personal knowledge or episodic memory (Szpunar, Spreng, \& Schacter, 2014). Moreover, because of this inherent difficulty in predicting how our values and preferences will change in the future, one may be misled to assume that such changes are unlikely (Quoidbach et al., 2013). This may explain why in the present study and in Renoult et al.'s study (2016), taking the current perspective of an older adult appears to result in the most accurate predictions, as neither preferences of participants nor those of their peers were thought to change enough in the future to be similar to those of older adults.

Common misconceptions about future changes can have severe consequences, such as making poor health or financial choices in the present that our future selves will regret. Attempting to think differently about our future may thus have far- reaching impact. Based on the successes of some interventions to change future predictions (Sherman et al., 1985; Szpunar \& Schacter, 2013), it is likely that reducing presentism biases is possible. Indeed, our results suggest that considering the current perspectives of generic peers or, even better, those of older adults, prior to making future simulations for oneself may lead to a more realistic estimation of the magnitude of likely changes in the future. 


\section{Appendix}

List of stimuli used in the perspective taking task

Stereotypically-young -person things:

1. Theme parks

2. Rollerblading

3. Converse shoes

4. Living in a busy city

5. White water rafting

6. Going clubbing

7. Going to festivals

8. Rollercoasters

9. Instagram

10. Late nights

Stereotypically-old-person things:

1. Playing chess

2. Dinner at 5

3. Marks and Spencer's department store

4. Volvo Cars

5. Living in the peaceful countryside

6. Birdwatching

7. Gardening

8. Cruise holidays

9. Knitting

10. Early nights 
References

Atance, C. M. (2008). Future thinking in young children. Current Directions in Psychological Science, 17(4), 295-298. doi: DOI 10.1111/j.1467-8721.2008.00593.x

Baker, L. A., \& Emery, R. E. (1993). When Every Relationship Is above-Average Perceptions and Expectations of Divorce at the Time of Marriage. Law and Human Behavior, 17(4), 439-450. doi: Doi 10.1007/Bf01044377

Bechara, A., \& Damasio, A. R. (2005). The somatic marker hypothesis: A neural theory of economic decision. Games and Economic Behavior, 52(2), 336-372. doi: DOI 10.1016/j.geb.2004.06.010

Cameron, J. J., Wilson, A. E., \& Ross, M. (2004). Autobiographical memory and selfassessment. In D. R. Beike, J. M. Lampinen \& D. A. Behrend (Eds.), The Self and Memory (pp. 207-226). New York: Psychology Press.

Chambers, J. R. (2008). Explaining False Uniqueness: Why We are Both Better and Worse Than Others. Social and Personality Psychology Compass, 2(2), 878-894

D'Argembeau, A., Renaud, O., \& Van der Linden, M. (2011). Frequency, Characteristics and Functions of Future-oriented Thoughts in Daily Life. Applied Cognitive Psychology, 25(1), 96-103. doi: Doi 10.1002/Acp.1647

Gilbert, D. T., Gill, M. J., \& Wilson, T. D. (2002). The future is now: Temporal correction in affective forecasting. Organizational Behavior and Human Decision Processes, 88(1), 430-444. doi: DOI 10.1006/obhd.2001.2982

Gilbert, D. T., \& Wilson, T. D. (2007). Prospection: Experiencing the future. Science, 317(5843), 1351-1354. doi: DOI 10.1126/science.1144161

Grysman, A., Prabhakar, J., Anglin, S. M., \& Hudson, J. A. (2013). The time travelling self: comparing self and other in narratives of past and future events. Conscious Cogn, 22(3), 742-755. doi: 10.1016/j.concog.2013.04.010

Jason, L. A., Schade, J., Furo, L., Reichler, A., \& Brickman, C. (1989). Time Orientation Past, Present, and Future Perceptions. Psychological Reports, 64(3), 1199-1205

Kanten, A. B., \& Teigen, K. H. (2008). Better than average and better with time: Relative evaluations of self and others in the past, present, and future. European Journal of Social Psychology, 38(2), 343-353. doi: 10.1002/ejsp.457

Kim, H., Schnall, S., \& White, M. P. (2013). Similar Psychological Distance Reduces Temporal Discounting. Personality and Social Psychology Bulletin, 39(8), 1005-1016. doi: $10.1177 / 0146167213488214$

Lee, W. S., \& Atance, C. M. (2016). The Effect of Psychological Distance on Children's Reasoning about Future Preferences. PLoS One, 11(10), e0164382. doi: 10.1371/journal.pone.0164382

Liberman, N., Trope, Y., \& Stephan, E. (2007). Psychological distance Social psychology: Handbook of basic principles (pp. 353-383). New York: Guilford Press.

Loewenstein, G. (1996). Out of control: Visceral influences on behavior. Organizational Behavior and Human Decision Processes, 65(3), 272-292. doi: DOI 10.1006/obhd.1996.0028

Loewenstein, G., O'Donoghue, T., \& Rabin, M. (2003). Projection bias in predicting future utility. Quarterly Journal of Economics, 118(4), 1209-1248. doi: Doi 10.1162/003355303322552784

Naqvi, N., Shiv, B., \& Bechara, A. (2006). The role of emotion in decision making: A cognitive neuroscience perspective. Current Directions in Psychological Science, 15(5), 260-264. doi: DOI 10.1111/j.1467-8721.2006.00448.x

Pronin, E., Olivola, C. Y., \& Kennedy, K. A. (2008). Doing unto future selves as you would do unto others: Psychological distance and decision making. Personality and Social Psychology Bulletin, 34(2), 224-236. doi: 10.1177/0146167207310023

Quoidbach, J., Gilbert, D. T., \& Wilson, T. D. (2013). The End of History Illusion. Science, 339(6115), 96-98. doi: DOI 10.1126/science.1229294 
Renoult, L., Kopp, L., Davidson, P. S., Taler, V., \& Atance, C. M. (2016). You'll change more than I will: Adults' predictions about their own and others' future preferences. Q J Exp Psychol (Hove), 69(2), 299-309. doi: 10.1080/17470218.2015.1046463

Sherman, S. J., Cialdini, R. B., Schwartzman, D. F., \& Reynolds, K. D. (1985). Imagining Can Heighten or Lower the Perceived Likelihood of Contracting a Disease - the Mediating Effect of Ease of Imagery. Personality and Social Psychology Bulletin, 11(1), 118-127. doi: Doi 10.1177/0146167285111011

Sui, J., \& Humphreys, G. W. (2015). The Integrative Self: How Self-Reference Integrates Perception and Memory. Trends Cogn Sci, 19(12), 719-728. doi: 10.1016/j.tics.2015.08.015

Szpunar, K. K., \& Schacter, D. L. (2013). Get Real: Effects of Repeated Simulation and Emotion on the Perceived Plausibility of Future Experiences. Journal of Experimental Psychology-General, 142(2), 323-327. doi: 10.1037/a0028877

Szpunar, K. K., Spreng, R. N., \& Schacter, D. L. (2014). A taxonomy of prospection: Introducing an organizational framework for future-oriented cognition. Proc Natl Acad Sci U S A. doi: 10.1073/pnas.1417144111

Tunney, R. J., \& Ziegler, F. V. (2015). Toward a Psychology of Surrogate Decision Making. Perspectives on Psychological Science, 10(6), 880-885. doi: $10.1177 / 1745691615598508$

Ziegler, F. V., \& Tunney, R. J. (2012). Decisions for Others Become Less Impulsive the Further Away They Are on the Family Tree. Plos One, 7(11). doi: ARTN e49479

10.1371/journal.pone.0049479 


\section{Figure Captions}

Figure 1: Average preference ratings. Mean preference rating for the self, close other and generic peer both 'now' and in the 'future', as well as the mean current preferences of an older adult. Stereotypically-young items are represented in blue and stereotypically-old items in orange. Error bars represent the $95 \%$ confidence intervals.

Figure 2: Change in preference ratings with time. Mean difference in preference ratings for the self, close other, and generic peer. These differences were obtained by subtracting future from current preferences (for the stereotypically-young items, in blue) and current from future preferences (for the stereotypically-old items, in orange). Error bars represent the $95 \%$ confidence intervals. 
Figure 1: Average preference ratings. Mean preference rating for the self, close other and generic peer both 'now' and in the 'future', as well as the mean current preferences of an older adult. Stereotypically-young items are represented in blue and stereotypically-old items in orange. Error bars represent the $95 \%$ confidence intervals.

$161 \times 88 \mathrm{~mm}(300 \times 300 \mathrm{DPI})$ 


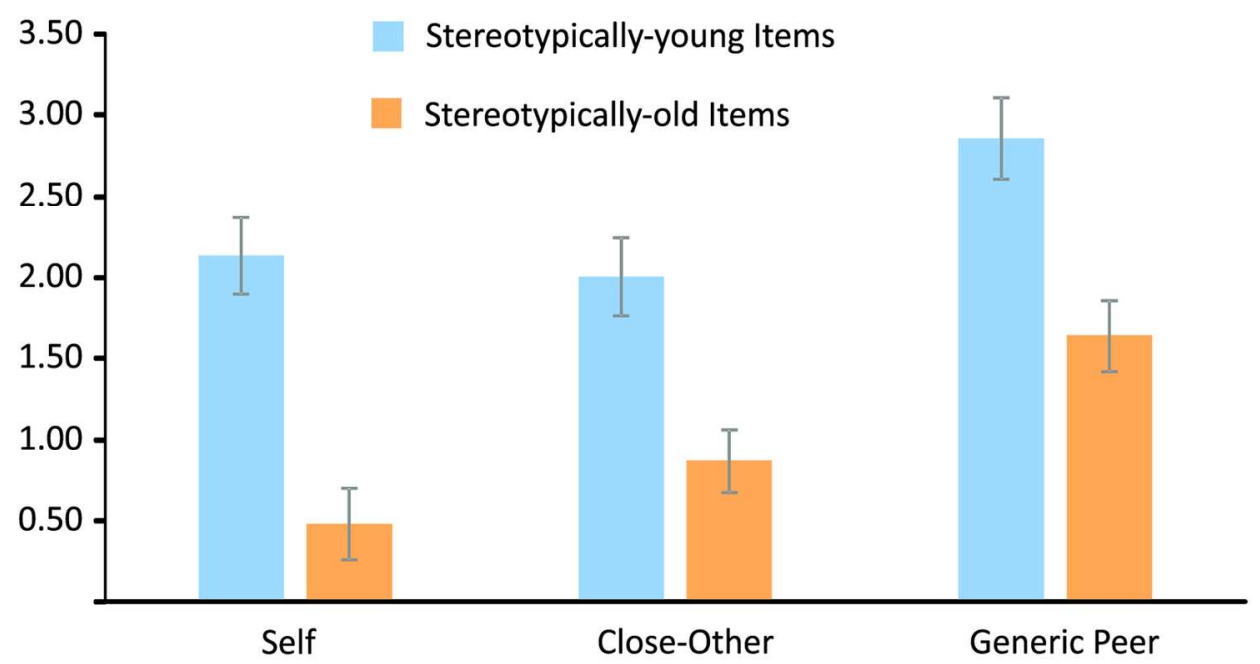

Figure 2: Change in preference ratings with time. Mean difference in preference ratings for the self, close other, and generic peer. These differences were obtained by subtracting future from current preferences (for the stereotypically-young items, in blue) and current from future preferences (for the stereotypically-old items, in orange). Error bars represent the $95 \%$ confidence intervals.

$$
142 \times 80 \mathrm{~mm}(300 \times 300 \mathrm{DPI})
$$

